\title{
Oral Health Status and Nutritional Habits as Predictors for Developing Alzheimer's Disease
}

\author{
Aleksandra Popovac ${ }^{a}$ Asja Čelebić ${ }^{b}$ Sanja Peršić ${ }^{b}$ Elka Stefanova ${ }^{c}$ \\ Aleksandra Milić Lemića Ivica Stančića \\ ${ }^{a}$ Clinic for Prosthodontics, School of Dental Medicine, University of Belgrade, Belgrade, Serbia; bepartment of \\ Removable Prosthodontics, School of Dental Medicine, University of Zagreb, Zagreb, Croatia; 'Clinic for Neurology, \\ Faculty of Medicine, University of Belgrade, Belgrade, Serbia
}

\section{Highlights of the Study}

- In addition to the number of a subject's teeth, the total number of functional tooth reduces a risk for developing Alzheimer's disease (AD).

- The associated risk factors for development of AD include poor oral health and nutritional status.

- This study suggests that oral health status, presence of functional tooth units, and nutritional status are interrelated.

\section{Keywords}

Alzheimer's disease · Oral health · Serum albumin · Food consistency

\footnotetext{
Abstract

Objective: Poor oral health, mainly tooth loss, has been suggested to pose a risk factor for Alzheimer's disease (AD). The nature of this relationship can be explained by mastication deficit and nutritional status. Also, the influence of nutritional parameters on cognitive impairment has been documented. The aim of this study was to investigate whether poor dental status and nutrition habits can be potential separate or associated risk factors for development of the AD. Methods: The study sample included 116 patients with AD and 63 controls. Sociodemographic variables were investigated as factors potentially associated with AD. Dental examination included recording the number of natural teeth, presence of fixed or removable dentures, and the number of functional
}

tooth units (FTUs). Nutritional status analysis included qualitative nutrition information, body mass index, serum albumin level, food consistency, and need for assistance in feeding. Regression analysis was used to investigate the predictorsfor development of AD. Results: Variables with significant differences between groups, which were analyzed by using the binary regression analysis, were marital status, residence, number of total FTUs (no matter whether the contacts were between natural teeth or dentures), eating meat/fish and fruits/vegetables, food consistency, and serum albumin level. Logistic regression analysis showed that being single/ widowed/divorced, eating more meat/fish or fruit/vegetable, eating blended/mashed/liquid food, having low levels of serum albumin, and having less FTUs were significant predictors for developing dementia. Conclusion: Having fewer occlusal contacts, consumption of soft food, and lower serum albumin levels can be considered as associated risk factors for AD. (c) 2021 The Author(s)

Published by S. Karger AG, Basel karger@karger.com www.karger.com/mpp

Karger $\stackrel{\text { ' }}{5}$

BOPEN ACCESS
(C) 2021 The Author(s)

Published by S. Karger AG, Basel

This is an Open Access article licensed under the Creative Common Attribution-NonCommercial-4.0 International License (CC BY-NC) (http://www.karger.com/Services/OpenAccessLicense), applicable to the online version of the article only. Usage and distribution for commercial purposes requires written permission.
Correspondence to:

Aleksandra Milić Lemić, aleksandra.milic@ @stomf.bg.ac.rs 


\section{Introduction}

Alzheimer's disease (AD) is the main cause of dementia and represents a significant challenge for health systems worldwide due to unreliable diagnosis, long-term functional dependence, and lack of effective medicaments. It is believed that the etiology of $\mathrm{AD}$ is multifactorial. The disease likely develops due to genetic factors, lifestyle, and environment [1]. Several studies suggest that poor oral health, especially tooth loss and periodontal disease, have negative impact on cognitive function [2-4]. One of the mechanisms that have been suggested to underlie association between poor oral health and dementia is nutrition, that is, compromised dentition affects quality of a diet and nutrient intake by leading to reliance on mashed food, carbohydrate, and confectioneries with too many calories, and fewer fruits and vegetables [5-7]. Many studies have investigated the association between nutrition and dementia, but the results are very diverse and do not support the recommendation of specific supplements, foods, or diets for the prevention of AD. Data suggest that the Mediterranean diet has a preventive role in $\mathrm{AD}$ due to monounsaturated fats and fatty acids from fish and vegetables, low in levels of saturated fat, and high in levels of antioxidants, such as vitamins $\mathrm{C}$ and $\mathrm{E}$, carotenoids, and flavonoids $[6,8]$. Nevertheless, other studies have not found any association between the Mediterranean diet and risk of developing dementia [9]. Also, caloric intake is mentioned as potential modulating factor for $\mathrm{AD}$, as increased in those with higher body mass index (BMI) variability [10] A systematic review suggested that vitamins B12, C, and $\mathrm{E}$ play an extensive role in the treatment and management of AD [11]. However, recent randomized controlled trials of various vitamins and supplements in general have not shown any statistically significant results [12]. Furthermore, some studies proved that chewing deficiency among the elderly was the most important variable explaining the differences in dietary intake $[5,13]$.

The aim of this study was to ascertain whether poor dental status and different nutrition habits can be potential separate or associated risk factors for development of the AD. The hypothesis is that worse oral health and nutritional status are associated risk factors for developing $\mathrm{AD}$.

\section{Methods}

\section{Subjects}

Two groups of participants were recruited for the study: $A D$ patients and control subjects without dementia. Inclusion criteria for the $\mathrm{AD}$ group was clinical diagnosis of probable $\mathrm{AD}$ based on the NINDS-ADRDA Alzheimer's Criteria [14] (other types of dementia were excluded), and for participants without dementia Mini-Mental State test scores $\geq 24$ [15]. For both groups, inclusion criteria were age 65 and more. In both groups, participants who had a significant change of dental status in the last 5 years (e.g., who received new dentures, or who had $>1$ tooth extracted, etc.) were excluded from the study. These criteria exist in order to investigate the potential influence of the existing dental status on the onset of dementia $[2,16]$, according to the case-control study design. All participants were recruited at the university hospital center and the gerontology center.

General characteristics of participants, such as gender, age, education, marital status, comorbidities (number of other chronic diseases besides $\mathrm{AD}$ ), and residence of patients (home or residential home) were registered. Interviews were conducted and written consents were obtained from patients' relatives or caregivers for the AD participants. The study was approved by the Ethics Committee of the School of Dental Medicine, University of Belgrade, with the decision number $36 / 18$. The study was conducted during the period November 2015-December 2017.

Study Design

Oral Health Status

The analysis of dental status was performed by a thorough dental examination. The obtained data were listed into different groups depending on a number of natural teeth $(<20, \geq 20)$, number of functional tooth units (FTUs), and presence of fixed or removable dentures (in edentulous and partially edentulous patients). Natural teeth and fixed dentures were placed in the same category. There were 2 groups for natural teeth: one was with $<20$ and the other with $>20$ [13]. The FTUs were determined depending on occluding pairs of opposing posterior natural teeth (i.e., sound, restored and D1-D4 scale carious teeth) or artificial teeth on implant-supported, fixed, and removable prostheses. D4 scale carious teeth with extensive coronal destruction and missing teeth were regarded as nonfunctional. Each of the opposing premolars was defined as 1 FTU, while each of the opposing molars was defined as 2 FTUs. The third molars were excluded from the FTU registration; thus, it was possible to register a maximum of 12 FTUs. Two categories of FTUs were included: a total number of FTUs (no matter whether opposing units were natural, fixed, or removable teeth) and a number of FTUs between natural teeth or fixed partial dentures [13]. The presence of prosthodontic appliances was recorded as no denture (in edentulous patients), presence of removable denture, and presence of fixed denture.

\section{Nutritional Habits}

Nutritional status analysis was carried out through conversation with participants or participants' family members or caregivers in cases of patients with $\mathrm{AD}$. Following categories were recorded: qualitative nutrition information, height, and weight of a patient, calculated BMI, serum albumin level, most frequent food consistency during meals and a need of assistance in feeding. Qualitative food intake represented frequency of consumption of $3 \mathrm{ba}$ sic food types - meat and/or fish, fruits and/or vegetables, and milk and/or dairy products. Every food type was grouped as often (4 times per week and more) or eating rarely (up to 3 times a week). According to the BMI values, participants were classified into 2 groups: (1) BMI $<21$ (representing a risk for malnutrition or malnutrition) and (2) BMI $>21$ (representing no risk for malnutri- 
Table 1. Sociodemographic data in patients with $\mathrm{AD}$ and controls

\begin{tabular}{|c|c|c|}
\hline Variable & $\begin{array}{l}\text { Dementia } \\
(n=116)\end{array}$ & $\begin{array}{l}\text { Without } \\
\text { dementia } \\
(n=63)\end{array}$ \\
\hline \multicolumn{3}{|l|}{ Gender, $n(\%)$} \\
\hline Male & $39(33.6)$ & $28(44.4)$ \\
\hline Female & $77(66.4)$ & $35(55.6)$ \\
\hline \multicolumn{3}{|l|}{ Marital status, $n(\%)$} \\
\hline Married & $62(53.4)$ & $47(74.6)$ \\
\hline Widowed/divorced/single & $54(46.6)$ & $16(25.4)$ \\
\hline \multicolumn{3}{|l|}{ Age, years } \\
\hline Mean \pm SD & $75.22 \pm 6.79$ & $76.9 \pm 6.57$ \\
\hline $95 \% \mathrm{CI}$ & $(73.98-76.47)$ & $(75.25-78.56)$ \\
\hline \multicolumn{3}{|l|}{ Residence, $n(\%)$} \\
\hline Home & $43(37.1)$ & $40(63.5)$ \\
\hline Institution & $73(62.9)$ & $23(36.5)$ \\
\hline \multicolumn{3}{|l|}{ Education, $n(\%)$} \\
\hline Primary school & $55(47.4)$ & $22(34.9)$ \\
\hline Medium school & $33(28.4)$ & $24(38.1)$ \\
\hline University degree & $28(24.1)$ & $17(27.0)$ \\
\hline \multicolumn{3}{|l|}{ Chronic diseases, $n(\%)$} \\
\hline None & $34(82.9)$ & $7(17.1)$ \\
\hline 1 or 2 & $62(60.2)$ & $41(39.8)$ \\
\hline 3 or more & $20(57.1)$ & $15(42.9)$ \\
\hline
\end{tabular}

$\mathrm{AD}$, Alzheimer's disease; SD, standard deviation; CI, confidence interval.

tion). Although malnutrition is defined as BMI below 19, some studies indicate that in elderly, especially nursing home residents, risk for malnutrition starts with values below $21[5,10]$. The serum albumin level was recorded and categorized into 2 groups: in the reference range $(35-52 \mathrm{~g} / \mathrm{L})$ or below the reference range. Food consistency was recorded as solid (firm, hard food) or soft (mashed, minced, and blended). Assistance in feeding was categorized as without assistance, with partial assistance, and with complete assistance.

\section{Statistical Analysis}

The statistical analysis was made with SPSS software (version 20.0; IBM Corp.). Descriptive statistics comprised calculation of frequencies for nominal variables, and arithmetic means and standard deviations for continuous or discrete variables. $\chi^{2}$ tests or independent $t$ tests were obtained to test the difference between the participants with and without $\mathrm{AD}$. Association between the variable dementia (yes, no) and all other variables was performed by point-bi-serial correlation or Spearman's rank correlation analysis. The binary logistic regression analysis was made to predict factors that may lead to dementia (which is a dichotomous binary variable). Dementia (yes, no) was entered as a dependent variable. Only variables (factors) with significant correlation coefficients with the dependent variable - dementia $(p<0.05)$ were entered into the model as independent variables (predictors). The omnibus tests were used to check whether the new model (with explanatory variables included) was an improvement over the baseline model. The Nagelkerke $R^{2}$ value was used to approximate how much vari-
Table 2. Sample description based on number of natural teeth, existence of prosthodontic appliances, and number of FTUs (no matter of the type of prosthodontic appliance)

\begin{tabular}{lll}
\hline Variable & $\begin{array}{l}\text { Dementia } \\
(n=116)\end{array}$ & $\begin{array}{l}\text { Without } \\
\text { dementia } \\
(n=63)\end{array}$ \\
\hline $\begin{array}{l}\text { Natural teeth, } n(\%) \\
\quad<20\end{array}$ & $96(82.8)$ & $55(87.3)$ \\
$\quad>20$ & $20(17.2)$ & $8(12.7)$ \\
FTUs, $n$ & $3.88 \pm 4.82$ & $9.10 \pm 4.60$ \\
$\quad \begin{array}{l}\text { Mean } \pm \text { SD } \\
\text { 95\% CI }\end{array}$ & $(2.99-4.77)$ & $7.94-10.25$ \\
FTU-NF), $n$ & $1.36 \pm 2.51$ & $1.48 \pm 2.85$ \\
$\quad$ Mean \pm SD & $(0.90-1.82)$ & $(0.75-2.19)$ \\
$\quad \begin{array}{l}\text { 95\% CI } \\
\text { Prosthodontic appliance, } n(\%)\end{array}$ & $61(84.7)$ & $11(15.3)$ \\
$\quad \begin{array}{l}\text { None edentulous } \\
\text { Removable denture }\end{array}$ & $22(81.5)$ & $5(18.5)$ \\
$\quad$ NF, n & $33(41.2)$ & $47(58.8)$ \\
\hline
\end{tabular}

$\mathrm{AD}$, Alzheimer's disease; SD, standard deviation; CI, confidence interval; FTUs, functional tooth units; FTU-NF, FTUs between natural teeth or fixed partial dentures; NF, natural teeth or fixed partial denture.

ance in the outcome was explained by the model (like $R^{2}$ in linear regression analysis). The Hosmer and Lemeshow test using the nonsignificance as the effect of goodness of fit was used to see the goodness of fit to the data. The level of significance for all statistical tests was set at 0.05 .

\section{Results}

A total of 179 participants were included in the study. The diagnosis of $\mathrm{AD}$ and dementia was established in 116 participants, while 63 of them represented control subjects without dementia. Sociodemographic data are shown in Table 1. Both groups were of similar age, and there was no significant difference in the level of education and gender. However, participants with a diagnosis of $\mathrm{AD}$ were more frequently single/widowed/divorced $(p=0.006)$, more frequently lived in institutions $(p=$ $0.001)$, and had fewer chronic diseases $(p=0.02)$.

Data obtained by clinical examination of dental status are presented in Table 2. The mean values of all FTUs were higher in the control subjects $(p<0.01)$; however, no significant differences were observed for the FTUs between natural teeth or fixed partial dentures, between the $\mathrm{AD}$ group and the control group. Both groups had more frequently fewer than 20 natural teeth, and there was no 
Table 3. Nutrition parameters in patients with $\mathrm{AD}$ and controls

\begin{tabular}{lcc}
\hline Variable & $\begin{array}{l}\text { Dementia } \\
(n=116), n(\%)\end{array}$ & $\begin{array}{l}\text { Without } \\
\text { dementia } \\
(n=63), n(\%)\end{array}$ \\
& & \\
\hline $\begin{array}{l}\text { Meat or fish } \\
\quad<3 \text { times a week }\end{array}$ & $59(50.9)$ & $44(69.8)$ \\
$\quad>4$ times a week & $57(49.1)$ & $19(30.2)$ \\
Fruit or vegetables & & \\
$\quad<3$ times a week & $23(19.8)$ & $22(34.9)$ \\
$\quad>4$ times a week & $93(80.2)$ & $41(65.1)$ \\
Milk and milk products & & \\
$\quad<3$ times a week & $41(35.3)$ & $21(33.3)$ \\
$\quad>4$ times a week & $75(64.7)$ & $42(66.7)$ \\
Food consistency & & \\
$\quad$ Solid food & $36(31.0)$ & $33(52.4)$ \\
$\quad$ Shredded, minced, or & & \\
$\quad$ blended food & $80(69.0)$ & $30(47.6)$ \\
Serum albumins & & \\
$\quad$ Below normal range & $39(33.6)$ & $9(14.3)$ \\
$\quad$ Normal range & $77(66.4)$ & $54(85.7)$ \\
BMI & & \\
$\quad<21$ & $59(50.9)$ & $28(44.4)$ \\
$\quad>21$ & $57(49.1)$ & $35(55.6)$ \\
Self-feeding or assistance during meals & \\
$\quad$ Self-feeding & $57(49.1)$ & $36(57.1)$ \\
Self-feeding and assistance & $27(23.3)$ & $7(11.1)$ \\
$\quad$ Complete assistance & $32(27.6)$ & $20(31.7)$ \\
\hline
\end{tabular}

$\mathrm{AD}$, Alzheimer's disease; BMI, body mass index.

significant difference between them. Patients with $\mathrm{AD}$ more frequently were edentulous without any dentures or had fixed partial dentures, while participants without AD more frequently had removable dentures $(p<0.001)$.

Parameters describing nutrition of the subjects are presented in Table 3. Half of the patients with dementia had a risk for malnutrition or were malnourished. There was no statistical difference between groups in terms of BMI. As regards qualitative nutritional status, there were significant differences in meat/fish $(p=0.018)$ and fruits/ vegetables consumption $(p=0.03)$, with $\mathrm{AD}$ subjects eating more frequently adequate food. Patients with $\mathrm{AD}$ more frequently consumed mashed food $(p=0.006)$. The serum albumin level was more frequently below normal range in patients with $\mathrm{AD}(p=0.005)$. Self-feeding was little more frequent in patients without dementia, but there was no significant difference between the groups.

The results of the logistic regression analysis are presented in Table 4. Variables with significant correlation coefficients were entered into the binary regression analysis: marital status, frequency of meat/fish, and fruit/vegetable consumption, the food consistency, serum albu-

Predictors for Developing Alzheimer's Disease mins, and number of total functional units. The Omnibus test revealed significance of the model coefficients $\left(\chi^{2}=\right.$ $78.72 ; \mathrm{df}=6 ; p<0.001)$. The Nagelkerke $R^{2}$ was 0.49 . The Hosner and Lemeshow test, using the nonsignificance as the effect of goodness of fit showed that the fit of the model was $\operatorname{good}\left(\chi^{2}=9.56, \mathrm{df}=8, p=0.298\right)$. The classification table showed $85.3 \%$ accuracy to predict dementia using the model, and $66.7 \%$ accuracy to predict those not having dementia. The overall accuracy was $78.8 \%$. The logistic regression equation is presented in Table 4 . The model equation described in Table 4 is as follows:

$$
\mathrm{Y}=-7.897+1.101 X 1+1.677 X 2+1.551 X 3+1.069 X 4+1.412 X 5-0.223 X 6
$$

Being single/widowed/divorced, eating more meat/ fish or fruit/vegetable, eating blended/mashed/liquid food, having low level of serum albumins, and having less functional units were significant predictors for developing dementia.

\section{Discussion}

The findings of the current study showed that being single, having less occlusal contacts (FTUs), eating mashed and soft food, eating more often meat/fish and fruits/vegetables, and having lower serum albumin level can be considered as predictors of AD. Eating soft food confers a 2.9-fold more chance to develop AD; having lower level of serum albumin raises likelihood of $\mathrm{AD}$ 4 -fold and being single 3 -fold. Having more occlusal posterior contacts decrease the chance of developing AD.

Although this study showed that eating more meat and fruits/vegetables increases the chance of developing $\mathrm{AD}$, these findings can be interpreted in 2 ways. Participants with $\mathrm{AD}$ have caregivers who take care of their diet, in special institutions or in their homes. In both cases caregivers tend to give them enough proteins, vitamins, and fibers. However, as participants with dementia more frequently had lower serum albumin levels, it is questionable how much proteins were absorbed from the swallowed food in the intestine. Also, some studies showed that frequent intake of animal fats and cholesterol can increase the risk of $\mathrm{AD}[6,17]$. In our study, caloric intake did not prove to be a risk factor, which is inconsistent with some literature data, suggesting that patients with $\mathrm{AD}$ have lower $\mathrm{BMI}$ due to worse chewing and swallowing ability and a need for assistance in feeding [18]. However, many authors suggested the $\mathrm{BMI}$ as a tool for nutritional screening, but it cannot replace comprehensive nutritional evaluation $[10,18]$. 
Table 4. Regression analysis of factors (variables) which were significantly correlated with the dependent variable - dementia (no, yes)

\begin{tabular}{lrrrrrrrr}
\hline Variables in the equation & \multirow{2}{*}{ B } & SE & Waid & df & $p$ value & Exp $(B)$ & $\begin{array}{l}\text { 95\% } \\
\text { lower }\end{array}$ & $\begin{array}{l}\text { C.I. for } \\
\text { Exp }(B) \text { upper }\end{array}$ \\
\hline Married/single or widowed & 1.101 & 0.44 & 6.160 & 1 & 0.013 & 3.006 & 1.260 & 7.171 \\
Meat/fish & 1.667 & 0.48 & 12.153 & 1 & $<0.001$ & 5.349 & 2.084 & 13.730 \\
Fruit/vegetable & 1.551 & 0.54 & 8.317 & 1 & 0.004 & 4.714 & 1.643 & 13.524 \\
Food consistency & 1.069 & 0.45 & 5.703 & 1 & 0.017 & 2.911 & 1.211 & 6.998 \\
Serum albumins & 1.412 & 0.58 & 5.900 & 1 & 0.015 & 4.104 & 1.313 & 12.825 \\
FTUs & -0.223 & 0.04 & 27.538 & 1 & $<0.001$ & 0.800 & 0.736 & 0.869 \\
Constant & -7.897 & 1.90 & 17.305 & 1 & $<0.001$ & $<0.000$ & & \\
\hline
\end{tabular}

Y, dementia; X1, married/single or widowed; X2, meat/fish; X3, fruit/vegetable; X4, food consistency; X5, serum albumins; X6, functional units. FTUs, functional tooth units; CI, confidence interval.

We observed that patients with $\mathrm{AD}$ were less likely to be married and to live in their own homes than healthy controls. Similar to our findings, researchers concluded in a study that elderly subjects without partners had higher chances to develop AD than those who were married [19]. It can be assumed that emotional factors of elderly people without partners or living in residential homes can lead to social problems and isolation, and act as a potential trigger for some diseases, including dementia.

In most studies, the number of teeth is used as a parameter for assessment of dental status, and numerous studies showed significantly lower number of teeth in patients with dementia which is not in accordance with our results. Some researchers concluded that having fewer than 11 teeth is a significant risk factor for AD development [20], while others indicated that healthy subjects with more teeth showed better results in cognitive tests [16]. In animal models, tooth loss has been related to structural brain differences, such as significantly lower total brain volume and gray matter volume [21]. Also, molar extraction and soft diet led to pathological changes in the hippocampus and cerebral cortex with a result in learning deficits $[22,23]$.

However, the number of a subject's teeth alone cannot be a representative of oral functioning, as teeth must have occlusal contacts to enable functional mastication. According to our findings, existence of FTUs and a higher number of FTUs reduced the risk for developing AD, no matter whether functional contacts were between natural teeth or removable denture teeth. Masticatory function with removable dentures is dependent on many factors, such as denture retention and stability;, comfort while chewing, and patient's adaptation. However, our findings showed that existence of posterior occlusal contacts, re- gardless of type, are important negative predictors of $\mathrm{AD}$. In support of this, a few studies on edentulous individuals indicated that oral rehabilitation with removable dentures lead to increase in regional cerebral blood volume, suggesting that mastication may be protective against brain degeneration and cognitive decline $[24,25]$. Also, in animal models, learning deficits were partially reversed when masticatory function was restored [22, 24]. Although most of these findings represent cross-sectional studies in which the cause-and-effect relationship is unclear, some recent studies objectively quantified masticatory performance using functional assessments and showed a relationship between decreased masticatory performance and dementia [26-30]. Moreover, neuroimaging studies in humans showed that brain regions linked to the memory and learning process are activated during strong chewing activities [24, 25].

Eating minced/blended food was a high positive predictor of falling into the target group of dementia. The upper and lower bounds of the 95\% confidence interval were above 1 in the binary regression for eating soft/ blended food, pointing out a strong relationship between the predictor and the target variable (dementia). Thus, food consistency may be a major justification for considering oral/dental and nutritional factors as associated predictors of $\mathrm{AD}$. The relationship between dental status and nutrition habits is well documented; furthermore, subjects with more teeth and FTUs had better chewing efficiency, leading us to conclude that they can eat more solid food [14]. Some findings have showed that the mean intakes of some key nutrients, such as carotene, vitamins A and C, milk and dairy products, and green-yellow vegetables, decreased with the increasing number of teeth lost, even among dentists, for whom sufficient dental care 
is available [5]. In another study, the consumption of vegetables, fish, and shellfish was significantly lower in those with ill-fitting dentures or compromised dentition with fewer posterior occlusal pairs [30]. This is at variance with our study which shows patients with AD had fewer FTUs but higher consumption of meat, fish, and vegetables. However, in that study, no significant differences were seen in dietary intake between those with well-fitting dentures and good natural dentition groups, which is in accordance our finding that occluding teeth pairs are more important oral health indicators than teeth number [30].

The limitations of this study include the small sample size, the retrospective study design, and methods of gathering information about nutritional habits. Studies identifying nutritional habits commonly use recalling methods which have some weaknesses and may not provide enough information to generalize about the individual's usual intake. Case-control studies cannot confirm the nature and direction of the complex relationship between health, nutrition, and dementia. Regardless, the results obtained in the present study may be a good starting point for further investigations in this field, in which additional nutritional parameters can be included to confirm this relationship and to understand it better.

\section{Conclusion}

Dental status and nutritional habits have showed significant relationship with $\mathrm{AD}$ occurrence. Having more occlusal contacts is more important as protective factor for $\mathrm{AD}$ than having more teeth, with no difference if contacts are from natural teeth or dentures. As regard nutritional factors, even though patients with $\mathrm{AD}$ consumed meat, fruits, and vegetables significantly more often, soft food consumption and low serum albumin level stood out as predictors of AD. Because of the recognized rela- tionship between occlusal contacts, food consistency and mastication, oral health factors, and nutritional habits can be considered as potential associated risk factors for $\mathrm{AD}$.

\section{Statement of Ethics}

Interviews were conducted, and written consents were obtained from relatives or caregivers of the $\mathrm{AD}$ participants. The study was approved by the Ethics Committee of the School of Dental Medicine, University of Belgrade, with the decision number $36 / 18$. The study was conducted during the period November 2015-December 2017.

\section{Conflict of Interest Statement}

The authors have no conflicts of interest to declare.

\section{Funding Sources}

This study was supported by the Ministry of Science project of bilateral science collaboration.

\section{Author Contributions}

All authors qualified for the authorship and made substantial contribution to the manuscript. A.P., A.M.L., and S.P. involved in the conception and design, A.P., A.M.L., and E.S. performed acquisition of data. A.P., A.C., and S.P. enrolled in analysis and interpretation of data; A.P. and A.M.L. in drafting the manuscript; I.S. and A.C. revising it critically for important intellectual content.

\section{Data Availability Statement}

The SPSS database has been uploaded.

\section{References}

1 Bird TD. Genetic factors in Alzheimer's disease. N Engl J Med. 2005;352(9):862-4.

2 Okamoto N, Morikawa M, Tomioka K, Yanagi M, Amano N, Kurumatani N. Association between tooth loss and the development of mild memory impairment in the elderly: the Fujiwara-kyo study. J Alzheimers Dis. 2015;44(3):777-86.

3 Lin JW, Chang CH, Caffrey JL. Examining the association between oral health status and dementia: a nationwide nested case-controlled study. Exp Biol Med. 2020 Feb;245(3):23144.
4 Tan ECK, Lexomboon D, Häbel H, Fastbom J, Eriksdotter M, Johnell K, et al. Xerogenic medications as a predictor for dental health intervention in people with dementia. J Alzheimers Dis. 2020;75(4):1263-71

5 Wakai K, Naito M, Naito T, Kojima M, Nakagaki $\mathrm{H}$, Umemura $\mathrm{O}$, et al. Tooth loss and intakes of nutrients and foods: a nationwide survey of Japanese dentists. Community Dent Oral Epidemiol. 2010;38:43-9.

6 Luchsinger JA, Mayeux R. Dietary factors and Alzheimer's disease. Lancet Neurol. 2004;3: 579-87.
7 Morris MC, Evans DA, Bienias JL Tangney CC, Bennett DA, Aggarwal N, et al. Dietary fats and the risk of incident Alzheimer disease. Arch Neurol. 2003;60:194200.

8 Scarmeas N, Stern Y, Tang MX, Mayeux R, Luchsinger JA. Mediterranean diet and risk for Alzheimer's disease. Ann Neurol. 2006;59: 912-21.

9 Pauwels EK. The protective effect of the Mediterranean diet: Focus on cancer and cardiovascular risk. Med Princ Pract. 2011;20:10311. 
10 Kang SY, Kim YJ, Jang W, Son KY, Park HS, Kim YS. Body mass index trajectories and the risk for Alzheimer's disease among older adults. Sci Rep. 2021 Feb 4;11(1):3087.

11 Silva LDS, Elemans VB, Luchsinger SJ, Kamphuis P, Yaffe K, Sijben J, et al. Plasma nutrient status of patients with Alzheimer's disease: systematic review and meta-analysis. Alzheimers Dement. 2014;10:485-502.

12 Bartochowski Z, Conway J, Wallach Y, Chakkamparambi B, Alakkasse S, Grossberg GT. Dietary interventions to prevent or delay Alzheimer's disease: what the evidence shows. Curr Nutr Rep. 2020;9:210-22.

13 Ueno M, Yanagisawa T, Shinada K, Ohara S, Kawaguchi Y. Category of functional tooth units in relation to the number of teeth and masticatory ability in Japanese adults. Clin Oral Investig. 2010;14:113-9.

14 McKhann G, Drachman D, Folstein M, Katzman R, Price D, Stadlan EM. Clinical diagnosis of Alzheimer's disease: report of the NINCDS-ADRDA Work Group under the auspices of department of health and human services task force on Alzheimer's disease. Neurology. 1984;34:939-44.

15 Folstein M, Folstein SE, McHugh PR. "Minimental state." A practical method of grading the cognitive state of patients for the clinician. J Psychiatr Res. 1975;12:189-98.

16 Takata Y, Ansai T, Soh I, Sonoki K, Awano S, Hamasaki T, et al. Cognitive function and number of teeth in a community-dwelling elderly population without dementia. J Oral Rehabil. 2009;36:808-13.
17 Sparks DL, Martins R, Martin T. Cholesterol and cognition: rationale for the $\mathrm{AD}$ cholesterol-lowering treatment trial and sex-related differences in beta amyloid accumulation in the brains of spontaneously hypercholesterolemic Watanabe rabbits. Ann N Y Acad Sci. 2002;977:356-66.

18 Bahat G, Tufan F, Saka B, Akin S, Ozkaya H, Yucel N, et al. Which body mass index (BMI) is better in the elderly for functional status? Arch Gerontol Geriatr. 2012;54(1):78-81.

19 Sundström A, Westerlund O, Mousavi-Nasab $H$, Adolfsson R, Nilsson LG. Relationship between marital and parental status and risk of dementia and Alzheimer's disease. Alzheimers Dement. 2013;9(4):631.

20 Arrivé E, Letenneur L, Matharan F, Laporte C Helmer C, Barberger-Gateau P, et al. Oral health condition of French elderly and risk of dementia: a longitudinal cohort study. Community Dent Oral Epidemiol. 2012;40(3): 230-8.

21 Dintica CS, Rizzuto D, Marseglia A, Kalpouzos G, Welmer AK, Wårdh I, et al. Tooth loss is associated with accelerated cognitive decline and volumetric brain differences: a population-based study. Neurobiol Aging. 2018; 67:23-30.

22 Watanabe K, Ozono S, Nishiyama K, Saito S, Tonosaki K, Fujita M, et al. The molarless condition in aged SAMP8 mice attenuates hippocampal Fos induction linked to water maze performance. Behav Brain Res. 2002; 128(1):19-25.

23 Ono Y, Kataoka T, Miyake S, Cheng SJ, Tachibana A, Sasaguri KI, et al. Chewing ameliorates stress-induced suppression of hippocampal long-term potentiation. Neuroscience. 2008;154(4):1352-9.
24 Miyamoto I, Yoshida K, Tsuboi Y, Iizuka T. Rehabilitation with dental prosthesis can increase cerebral regional blood volume. Clin Oral Implants Res. 2005;16(6):723-7.

25 Narita N, Kamiya K, Yamamura K, Kawasaki S, Matsumoto T, Tanaka N. Chewing related prefrontal cortex activation while wearing partial denture prosthesis: pilot study. Prosthodont Res. 2009;53(3):126-35.

26 Kim EK, Lee SK, Choi YH, Tanaka M, Hirotsu K, Kim HC, et al. Relationship between chewing ability and cognitive impairment in the rural elderly. Arch Gerontol Geriatr. 2017; 70:209-13.

27 Kimura Y, Ogawa H, Yoshihara A, Yamaga T, Takiguchi T, Wada T, et al. Evaluation of chewing ability and its relationship with activities of daily living, depression, cognitive status and food intake in the communitydwelling elderly. Geriatr Gerontol Int. 2013; 13(3):718-25.

28 Elsig F, Schimmel M, Duvernay E, Giannelli SV, Graf CE, Carlier S, et al. Tooth loss, chewing efficiency and cognitive impairment in geriatric patients. Gerodontology. 2015;32(2): 149-56.

29 Weijenberg RA, Lobbezoo F, Visscher CM, Scherder EJ. Oral mixing ability and cognition in elderly persons with dementia: a crosssectional study. J Oral Rehabil. 2015;42(7): 481-6.

30 Iwasaki M, Taylor GW, Manz MC, Yoshihara A, Sato M, Muramatsu K, et al. Oral health status: relationship to nutrient and food intake among 80-year-old Japanese adults. Community Dent Oral Epidemiol. 2014;42: 441-50. 